\title{
Predicting response of bladder cancers to gemcitabine and carboplatin neoadjuvant chemotherapy through genome-wide gene expression profiling
}

\author{
YOICHIRO KATO ${ }^{1,2}$, HITOSHI ZEMBUTSU ${ }^{1}$, RYO TAKATA ${ }^{2}$, FUYUKI MIYA ${ }^{3}$, TATSUHIKO TSUNODA ${ }^{3}$, \\ WATARU OBARA ${ }^{2}$, TOMOAKI FUJIOKA ${ }^{2}$ and YUSUKE NAKAMURA ${ }^{1}$ \\ ${ }^{1}$ Laboratory of Molecular Medicine, Human Genome Center, Institute of Medical Science, The University of Tokyo, \\ Tokyo 108-8639; ${ }^{2}$ Department of Urology, Iwate Medical University, Morioka 020-8505; \\ ${ }^{3}$ Laboratory for Medical Informatics, Center for Genomic Medicine, RIKEN, Yokohama 230-0045, Japan
}

Received October 20, 2010; Accepted November 4, 2010

DOI: $10.3892 /$ etm.2010.166

\begin{abstract}
Neoadjuvant chemotherapy with gemcitabine and carboplatin (GC) for invasive bladder cancer increases the chance of a radical response for a subset of patients, while other patients suffer from severe adverse drug reactions without any benefit. To establish a method for predicting the response to chemotherapy with GC, the expression profiles of biopsy samples from 37 advanced bladder cancers were analyzed using a microarray consisting of 38,500 genes or ESTs. Upon analysis of 9 'responder' and 9 'non-responder' tumors, 12 'predictive' genes were found to be significantly differentially expressed between the 'responder' and 'nonresponder' groups, and a numerical prediction scoring system that clearly separated the responder group from the non-responder group was established. This system accurately predicted the drug responses of 18 of 19 additional test cases that were reserved from the original 37 cases. Moreover, a quantitative PCR-based prediction system was developed that may be feasible for routine clinical use, and the sensitivity of invasive bladder cancer to neoadjuvant chemotherapy with GC was able to be predicted by the expression patterns in this set of genes. Nearly $50 \%$ of patients treated with GC or methotrexate, vinblastine, doxorubicin and cisplatin (M-VAC) therapy have been reported to achieve complete or partial response to either
\end{abstract}

Correspondence to: Dr Yusuke Nakamura, Laboratory of Molecular Medicine, Human Genome Center, Institute of Medical Science, The University of Tokyo, 4-6-1 Shirokanedai, Minato-ku, Tokyo 108-8639, Japan

E-mail: yusuke@ims.u-tokyo.ac.jp

Abbreviations: GC, gemcitabine and carboplatin; M-VAC, methotrexate, vinblastine, doxorubicin and cisplatin; PR, partial response; PS, performance status; AUC, area under the curve; RT-PCR, reverse transcription-polymerase chain reaction

Key words: gemcitabine, carboplatin, bladder cancer, neoadjuvant chemotherapy, prediction, gene expression profile, microarray of these therapies. When we applied this prediction system as well as the system for M-VAC, we expected that approximately $80 \%$ of the patients would achieve significant tumor shrinking (>60\%) by selection of either the GC or M-VAC regimens. Our results suggest that the two prediction scoring systems lead to achievement of 'personalized therapy' for the treatment of invasive bladder cancer and should improve the quality of life for patients with this disease.

\section{Introduction}

Bladder cancer is the second most common genitourinary tumor, having an incidence of 357,000 new cases each year worldwide; approximately one-third of these cases are likely to be invasive or metastatic disease at the time of diagnosis (1). Although radical cystectomy is considered as the gold standard for the treatment of patients with localized, but muscle-invasive, bladder cancer, nearly $50 \%$ of patients develop metastases within 2 years after cystectomy and subsequently die of the disease (2).

Neoadjuvant chemotherapy is usually applied to muscleinvasive bladder cancer to manage micrometastases and improve prognosis $(3,4)$. A neoadjuvant chemotherapy regimen involving gemcitabine and carboplatin (GC) as well as that of methotrexate, vinblastine, doxorubicin and cisplatin (M-VAC), followed by radical cystectomy, have been found to decrease the recurrence rate compared to radical cystectomy alone and to improve survival (5-8). Furthermore, a small subset of patients who respond well to neoadjuvant chemotherapy may have a chance to preserve bladder function and maintain a good quality of life.

However, since no method yet exists for predicting the response of an individual patient to GC therapy, some patients suffer from adverse reactions to these drugs without achieving any benefit in terms of a positive effect. They often are not able to undergo additional treatment as their physical condition deteriorates $(9,10)$. Hence, the development of a reliable method to predict the effectiveness of a specific therapy is critical to assign the appropriate treatment to individual patients with bladder cancer. Various factors have 
been reported to be associated with chemosensitivity or prognosis, yet each of these factors is not sufficiently effective to reliably predict individual response. Since cancer cells in individual patients are characterized by a large number of features, a larger body of information is required to precisely characterize them. The profiling of gene expression patterns on genome-wide microarrays enables investigators to perform comprehensive analyses of complex molecular activities in cancer cells. Systematic analysis of the expression levels of thousands of genes is also a useful approach for identifying molecules related to response to chemotherapy or radiation therapy.

In this study, we report the establishment of a system for predicting response to GC neoadjuvant chemotherapy for patients with invasive bladder cancer using genome-wide information obtained for 37 cases using a microarray consisting of 38,500 genes. This was carried out in combination with laser microbeam microdissection of the tumors to enrich the proportion of cancer cells for accurate analysis. We identified 12 genes that exhibited significantly different levels of expression between responders and non-responders who received a neoadjuvant GC regimen. In addition, although the response rate to each of the M-VAC and GC regimens was reported to be nearly $50 \%$, we suggest that the personalized selection of an appropriate chemotherapy regime using prediction systems for the response to these regimens may improve the response rate to approximately $80 \%$. Our results strongly imply that 'personalized therapy' based on expression levels of a small number of genes may improve the quality of life of a larger proportion of patients with invasive bladder cancer.

\section{Materials and methods}

Patients, tissue samples and neoadjuvant chemotherapy. Bladder cancer tissue samples from punch biopsy and corresponding clinical information were obtained from the Iwate Medical University after each patient provided written informed consent. A total of 37 cancer samples from patients (6 females and 31 males; median age, 67 years; age range, 52-78 years) (Table I) histologically confirmed as having transitional cell carcinoma of the bladder were selected for this study. The clinical stage of each patient was judged according to the International Union Against Cancer Tumor-NodeMetastasis classification; we enrolled only patients who had no node metastasis at clinical stages T2aNOM0 to T4aNOM0 and were expected to undergo radical cystectomy without prior radiation therapy. All participants had no serious abnormality in renal, hepatic or hematologic function and an Eastern Cooperative Oncology Group performance status (PS) judged to be $\leq 2$. Three to five pieces of cancer tissue were obtained from each patient at the time of biopsy before neoadjuvant chemotherapy. These samples were immediately embedded in TissueTek OCT compound (Sakura, Tokyo, Japan), frozen and stored at $-80^{\circ} \mathrm{C}$. The frozen tissues were sliced into $8-\mu \mathrm{m}$ sections using a cryostat (Sakura) and were then stained with $\mathrm{H} \& \mathrm{E}$ for histologic examination. Bladder cancer cells were selectively enriched for our experiments using the EZ-cut system with a pulsed UV narrow beam focus laser (SL Microtest GmbH, Germany) according to the manufacturer's protocols. All patients were examined through chest X-ray, computed tomography and magnetic resonance imaging of their abdomen and pelvis and were confirmed to have neither lymph node nor distant metastases. Patients were administered two 28-day cycles of GC neoadjuvant chemotherapy as follows: gemcitabine $\left(1,000 \mathrm{mg} / \mathrm{m}^{2}\right)$ on days 1,8 and 15 ; carboplatin (5 AUC) on day 2. According to their responses to the treatment, we categorized the patients into two groups: 'responders' who achieved significant tumor shrinking $(>60 \%)$ after two courses of GC neoadjuvant chemotherapy, and 'non-responders' who revealed no significant tumor shrinking $(\leq 60 \%)$ after the two courses of chemotherapy.

GeneChip hybridization. The Affymetrix human genome U133 Plus 2.0 GeneChip arrays were used for microarray hybridizations. This GeneChip comprises $>54,000$ probe sets and analyzes the expression level of 47,400 transcripts. For microarray hybridization, we followed the protocol described in the Affymetrix GeneChip 3'IVT Express Kit User Manual Protocol (Affymetrix, Santa Clara, CA, USA). For the synthesis of single-strand cDNA, 30-150 ng of total RNA was reversely transcribed using the First-Strand Enzyme mix and Buffer mix included in the GeneChip 3'IVT Express kit and double-strand cDNA synthesis according to the manufacturer's instructions followed by the Second-Strand Enzyme mix and Buffer mix included in the GeneChip 3'IVT Express kit. IVT amplification generates multiple copies of biotin-modified aRNA from the double-stranded cDNA templates (Affymetrix). A 15- $\mu \mathrm{g}$ aliquot of the labeled product was fragmented by heat and ion-mediated hydrolysis at $94^{\circ} \mathrm{C}$ for $35 \mathrm{~min}$ in $\mathrm{H}_{2} \mathrm{O}$ and $8 \mu \mathrm{l}$ of $5 \mathrm{X}$ fragmentation buffer (Affymetrix). The fragmented cRNA was hybridized for $16 \mathrm{~h}$ at $45^{\circ} \mathrm{C}$ in a Hybridization Oven 640 to a U133 Plus 2.0 oligonucleotide array (Affymetrix). The washing and staining of the arrays with phycoerythrin-conjugated streptavidin (Molecular Probes, Eugene, OR, USA) was completed in a Fluidics Station 450 (Affymetrix). The arrays were then scanned using a confocal laser GeneChip scanner 3000 (Affymetrix).

Data analysis. The obtained image files were analyzed with the Affymetrix data suite system, Microarray Suite 5.0 (MAS 5.0; Affymetrix). Global normalization at a target value of 500 was applied to all 38 arrays (37 cancer arrays and one array of the universal control) using the Affymetrix ${ }^{\circledR}$ GeneChip ${ }^{\circledR}$ Command Console 1.1 (Affymetrix). Normalized data from text files were imported to a Microsoft Excel spread sheet. Since data derived from low-signal intensities are less reliable, we excluded transcripts with low intensities from further analysis when the signal intensities of both the normal and cancer cells were lower than that of the cut-off. For the other genes, we calculated the signal intensities of the cancer/ normal ratio using the raw data of each sample (11).

Hierarchical clustering analysis. We used web-available software ('Cluster' and 'TreeView') written by M. Eisen (http:// genome-www5 .stanford.edu/MicroArray/SMD/restech.html) to create a graphic representation of the microarray data and to create a dendrogram of hierarchical clustering. Before the clustering algorithm was applied, the fluorescence ratio for each spot was first log-transformed, and then the data for each sample were median-centered to remove experimental biases. 
Table I. Clinicopathological features of the examined bladder cancer patients.

\begin{tabular}{|c|c|c|c|c|c|c|c|}
\hline ID no. & Gender & Age & Stage & Grade & Response & Prediction & Post treatment \\
\hline BCGC1006 & M & 60 & $\mathrm{~T} 4$ & G3 & Responder & Learning & Cystectomy \\
\hline BCGC1007 & $\mathrm{F}$ & 71 & $\mathrm{~T} 3 \mathrm{a}$ & $\mathrm{G} 2$ & Responder & Learning & Cystectomy \\
\hline BCGC1010 & M & 67 & T3b & G3 & Responder & Learning & Cystectomy \\
\hline BCGC1011 & M & 69 & T3b & - & Responder & Learning & Cystectomy \\
\hline BCGC1016 & M & 53 & T3a & G2 & Responder & Learning & Cystectomy \\
\hline BCGC1020 & $\mathrm{F}$ & 62 & $\mathrm{~T} 2 \mathrm{a}$ & G3 & Responder & Learning & Cystectomy \\
\hline BCGC1021 & M & 67 & T3a & G2 & Responder & Learning & Cystectomy \\
\hline BCGC1022 & M & 71 & $\mathrm{~T} 2 \mathrm{a}$ & G3 & Responder & Learning & Cystectomy \\
\hline BCGC1029 & M & 56 & T3b & G3 & Responder & Learning & Cystectomy \\
\hline BCGC1005 & M & 56 & $\mathrm{~T} 2 \mathrm{~b}$ & G1 & Non-responder & Learning & Cystectomy \\
\hline BCGC1009 & M & 60 & $\mathrm{~T} 4$ & G3 & Non-responder & Learning & Radiation \\
\hline BCGC1015 & M & 78 & T3a & $\mathrm{G} 2$ & Non-responder & Learning & Cystectomy \\
\hline BCGC1017 & M & 59 & T3a & G3 & Non-responder & Learning & Cystectomy \\
\hline BCGC1018 & M & 72 & $\mathrm{~T} 3 \mathrm{a}$ & $\mathrm{G} 2$ & Non-responder & Learning & Cystectomy \\
\hline BCGC1024 & M & 67 & $\mathrm{~T} 2 \mathrm{a}$ & $\mathrm{G} 2$ & Non-responder & Learning & Cystectomy \\
\hline BCGC1025 & M & 52 & $\mathrm{~T} 2 \mathrm{~b}$ & G3 & Non-responder & Learning & Radiation \\
\hline BCGC1026 & M & 62 & $\mathrm{~T} 4$ & G3 & Non-responder & Learning & Radiation \\
\hline BCGC1027 & M & 62 & T3b & G3 & Non-responder & Learning & Radiation \\
\hline BCGC1001 & M & 60 & $\mathrm{~T} 4$ & G1 & Responder & Test & Cystectomy \\
\hline BCGC1003 & M & 64 & $\mathrm{~T} 3 \mathrm{~b}$ & G3 & Responder & Test & Cystectomy \\
\hline BCGC1012 & M & 67 & $\mathrm{~T} 2 \mathrm{a}$ & G3 & Responder & Test & Cystectomy \\
\hline BCGC1013 & M & 74 & T3b & G2 & Responder & Test & Cystectomy \\
\hline BCGC1014 & M & 76 & T3b & $\mathrm{G} 2$ & Responder & Test & Cystectomy \\
\hline BCGC1019 & $\mathrm{F}$ & 71 & $\mathrm{~T} 2 \mathrm{a}$ & G2 & Responder & Test & TUR-Bt \\
\hline BCGC1031 & M & 75 & $\mathrm{~T} 2 \mathrm{a}$ & G3 & Responder & Test & Cystectomy \\
\hline BCGC1033 & $\mathrm{F}$ & 71 & $\mathrm{~T} 2 \mathrm{a}$ & $\mathrm{G} 2$ & Responder & Test & Cystectomy \\
\hline BCGC1036 & M & 71 & T3b & $\mathrm{G} 2$ & Responder & Test & Cystectomy \\
\hline BCGC1041 & M & 68 & $\mathrm{~T} 2 \mathrm{~b}$ & G3 & Responder & Test & Cystectomy \\
\hline BCGC1045 & M & 67 & $\mathrm{~T} 2 \mathrm{~b}$ & G3 & Responder & Test & GCx1 \\
\hline BCGC1030 & M & 55 & $\mathrm{~T} 2 \mathrm{~b}$ & G2 & Non-responder & Test & Cystectomy \\
\hline BCGC1032 & M & 70 & $\mathrm{~T} 2 \mathrm{a}$ & G3 & Non-responder & Test & Radiation \\
\hline BCGC1034 & $\mathrm{F}$ & 59 & $\mathrm{~T} 2 \mathrm{a}$ & $\mathrm{G} 2$ & Non-responder & Test & - \\
\hline BCGC1037 & M & 66 & $\mathrm{~T} 2 \mathrm{a}$ & G2 & Non-responder & Test & - \\
\hline BCGC1039 & M & 73 & T3a & G2 & Non-responder & Test & - \\
\hline BCGC1047 & M & 71 & $\mathrm{~T} 4$ & G3 & Non-responder & Test & Radiation \\
\hline BCGC1048 & M & 74 & T3b & G2 & Non-responder & Test & GCx1 \\
\hline BCGC1049 & $\mathrm{F}$ & 70 & $\mathrm{~T} 4$ & G3 & Non-responder & Test & GCx1 \\
\hline
\end{tabular}

Response, response to neoadjuvant GC treatment. Responder, patient who achieved significant tumor shrinking (>60\%) after two courses of GC neoadjuvant chemotherapy; Non-responder, patient who did not achieve significant shrinking of the tumors $(\leq 60 \%)$ after two courses of chemotherapy. Learning, samples used to develop the prediction system. Test, samples used for test cases. TUR-Bt, transurethral resection of the bladder tumor.

Identification of discriminating genes for chemosensitivity. We applied a random permutation test to identify genes that were expressed at a significantly different level between the two groups; that is, tumors with good response and those with poor response to the chemotherapy. Mean $(\mu)$ and standard deviation $(\delta)$ were calculated from the log-transformed relative expression ratios of each gene in the responder (r) and non-responder (n) cases. A discrimination score (DS) for each gene was defined as follows: DS $=\left(\mu_{\mathrm{r}}-\mu_{\mathrm{n}}\right) /\left(\delta_{\mathrm{r}}+\delta_{\mathrm{n}}\right)$.
We carried out permutation tests to estimate the ability of these individual genes to distinguish between responders and non-responders; samples were randomly permutated between the two groups, 10,000 times. Since the DS data set of each gene showed a normal distribution, we calculated a P-value for the user-defined grouping (12). For the initial analysis, we applied the expression data obtained for the 18 cases (9 responders and 9 non-responders) that were obtained at an earlier stage of the study. 


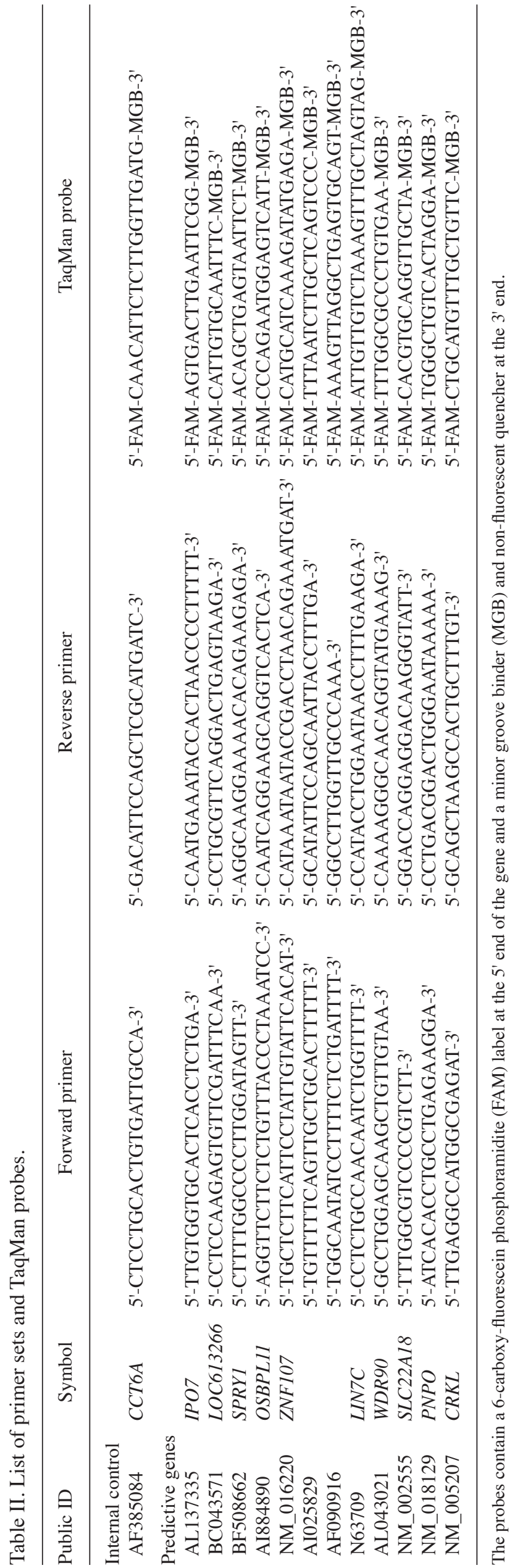

Calculation of the prediction score. We calculated the prediction scores (PS) according to procedures described previously (12). Each gene (gi) votes for either responder or non-responder depending on whether the expression level $\left(\mathrm{x}_{\mathrm{i}}\right)$ in the sample is closer to the mean expression level of the responders or non-responders in the reference samples. The magnitude of the vote $\left(\mathrm{V}_{\mathrm{i}}\right)$ reflects the deviation of the expression level in the sample from the average of the two classes: $V_{i}=1 x_{i}-\left(\mu_{\mathrm{r}}\right.$ $\left.+\mu_{\mathrm{n}}\right) / 21$.

We summed the votes to obtain total votes for the responders $\left(\mathrm{V}_{\mathrm{r}}\right)$ and non-responders $\left(\mathrm{V}_{\mathrm{n}}\right)$ and calculated PS values as follows: $P S=\left[\left(V_{r}-V_{n}\right) /\left(V_{r}+V_{n}\right)\right] \times 100$, reflecting the margin of victory in the direction of either responder or non-responder. PS values range from -100 to 100; a higher absolute value of PS reflects a stronger prediction.

Evaluation of the classification and leave-one-out method. We calculated the classification score (CS) using prediction scores of the responders $\left(\mathrm{PS}_{\mathrm{r}}\right)$ and non-responders $\left(\mathrm{PS}_{\mathrm{n}}\right)$ in each gene set as follows: $\mathrm{CS}=\left[\mu\left(\mathrm{PS}_{\mathrm{r}}\right)-\mu\left(\mathrm{PS}_{\mathrm{n}}\right)\right] /\left[\delta\left(\mathrm{PS}_{\mathrm{r}}\right)+\right.$ $\left.\delta\left(\mathrm{PS}_{\mathrm{n}}\right)\right]$.

A larger value of CS indicates better separation of the two groups by the prediction scoring system. For the leaveone-out test, one sample is withheld, the permutation P-value and mean expression levels are calculated using the remaining samples and the class of the withheld sample is subsequently evaluated by calculating its prediction score. We repeated this procedure for each of the 18 samples.

Quantitative reverse transcription-PCR. Aliquots of the same aRNA hybridized to the microarray from individual samples were reversely transcribed using random hexamer and SuperScript II reverse transcriptase (Invitrogen, Carlsbad, CA, USA). The expression of the 12 predictive genes and 1 endogenous control gene was measured by quantitativeRT-PCR using TaqMan Gene Expression Assay products on a Light Cycler 480 system (Roche Applied Science, Basel, Switzerland) as described previously $(13,14)$. The sequences of the primers and fluorogenic TaqMan MGB probes are listed in Table II. To normalize the expression of each gene, we selected as internal controls chaperonin-containing TCP1, subunit 6A (CCT6A), since this showed the smallest fluctuations of cancer/normal ratio in our bladder cancer microarray data as described previously (13). For the generation of standard curves we used a mixture of mRNAs derived from the bladder cancer samples. Quantitative RT-PCR experiments were performed in duplicate for all 12 predictive genes, and the relative expression ratios of each sample were calculated. The normalized gene expression values were log-transformed (on a base 2 scale) in a manner similar to the transformation of the microarray-based hybridization data.

Simulation of response of GC- or MVAC-treated patients to $M$-VAC or GC therapy. To simulate the clinical response of GC- or MVAC-treated patients to M-VAC or GC therapy, we first estimated the accuracies of the prediction scoring systems: positive and negative prediction accuracies (PPA and NPA) for test cases with positive and negative prediction scores, respectively. We calculated the accuracy of each of the prediction scoring systems for $\mathrm{GC}\left(\mathrm{PPA}_{\mathrm{GC}}, \mathrm{NPA}_{\mathrm{GC}}\right)$ and 
Table III. List of 14 discriminating genes.

\begin{tabular}{|c|c|c|c|c|}
\hline Public ID & Gene symbol & Gene name & P-value & Group $^{b}$ \\
\hline AL137335 & IPO7 & Importin 7 & $3.96 \times 10^{-7}$ & - \\
\hline ВC043571 & LOC613266 & Hypothetical LOC613266 & $8.38 \times 10^{-7}$ & + \\
\hline BF508662 & $S P R Y 1$ & Sprouty homolog 1, antagonist of FGF signaling (Drosophila) & $5.34 \times 10^{-6}$ & - \\
\hline AI884890 & OSBPLI1 & Oxysterol binding protein-like 11 & $1.81 \times 10^{-5}$ & + \\
\hline NM_016220 & ZNF107 & Zinc finger protein 107 & $1.96 \times 10^{-5}$ & + \\
\hline AI025829 & & CDNA clone IMAGE:5287121 & $2.45 \times 10^{-5}$ & - \\
\hline AF090916 & & Clone HQ0312 & $2.90 \times 10^{-5}$ & + \\
\hline N63709 & LIN7C & Lin-7 homolog C (C. elegans) & $3.18 \times 10^{-5}$ & - \\
\hline AL043021 & WDR90 & WD repeat domain 90 & $3.19 \times 10^{-5}$ & + \\
\hline NM_002555 & $S L C 22 A 18$ & Solute carrier family 22, member 18 & $3.30 \times 10^{-5}$ & - \\
\hline NM_018129 & $P N P O$ & Pyridoxamine 5'-phosphate oxidase & $4.75 \times 10^{-5}$ & - \\
\hline NM_005207 & $C R K L$ & V-crk sarcoma virus CT10 oncogene homolog (avian)-like & $6.92 \times 10^{-5}$ & + \\
\hline AL050145 & & Transcribed locus & $7.78 \times 10^{-5}$ & + \\
\hline BG284709 & LOC283871 & Hypothetical protein LOC283871 & $9.11 \times 10^{-5}$ & + \\
\hline
\end{tabular}

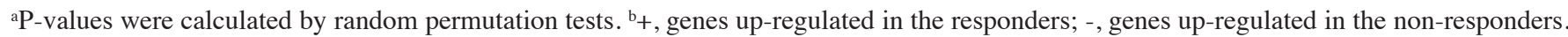
Information was retrieved from the Unigene database of the National Center for Biotechnology Information (build \#159).

M-VAC (PPA $\left.{ }_{\text {MVAC }}, \mathrm{NPA}_{\mathrm{MVAC}}\right)$ therapies, respectively. Among the 19 test cases (Fig. 2B) for the GC therapy, all of the 10 cases with positive scores were actually responders. Eight of the 9 cases with negative scores were non-responders. Therefore, the positive and negative predictive accuracies of this system were $\mathrm{PPA}_{\mathrm{GC}}=10 / 10$ and $\mathrm{NPA}_{\mathrm{GC}}=8 / 9$. However, in our previous study among the 21 test cases for the prediction scoring system for the M-VAC therapy, 14 of 16 cases with positive prediction scores were responders and the remaining 2 cases were non-responders (data not shown). All of the 5 cases with negative prediction scores were non-responders (data not shown). Therefore, the positive and negative predictive accuracies were $\mathrm{PPA}_{\mathrm{MVAC}}=14 / 16$ and $\mathrm{NPA}_{\mathrm{MVAC}}=5 / 5$.

Based on these results, we re-estimated the number of simulated GC/M-VAC responders: the number of estimated non-responders using both the GC and M-VAC prediction scoring systems was, based on the initial 13 cases (Fig. 4A-C), $13 \times \mathrm{NPA}_{\mathrm{GC}} \times \mathrm{NPA}_{\mathrm{MVAC}}$. Also, among the 29 cases predicted to be GC non-responders, but M-VAC responders, $29 \times \mathrm{NPA}_{\mathrm{GC}} \mathrm{X}$ (1 - $\left.\mathrm{PPA}_{\mathrm{MVAC}}\right)$ cases should be reconsidered as non-responders to both GC and M-VAC. Therefore, of the 76 cases in total, the number of estimated GC/M-VAC responders was calculated to be: $76-\left[13 \times \mathrm{NPA}_{\mathrm{GC}} \times \mathrm{NPA}_{\mathrm{MVAC}}+29 \times \mathrm{NPA}_{\mathrm{GC}} \mathrm{x}\right.$ $\left.\left(1-\mathrm{PPA}_{\mathrm{MVAC}}\right)\right]=61.2$.

\section{Results}

Identification of genes associated with GC neoadjuvant chemotherapy for bladder cancer. We enrolled 37 patients with invasive bladder cancer whose clinicopathological features are summarized in Table I. We defined patients who achieved significant tumor shrinking $(>60 \%)$ after two courses of GC neoadjuvant chemotherapy as 'responders', as we used the definition for the establishment of the prediction score for response to M-VAC therapy $(13,15)$. We compared the microarray expression profiles of tumors from the 9 responders and 9 non-responders and identified a set of genes that distinguished the two groups in accordance with the following criteria: signal intensities higher than the cut-off level in $>60 \%$ of samples of at least one group. Then, we carried out a random permutation test to select genes that may be associated with the drug response (see Materials and methods). We identified 14 genes that showed permutation P-values of $<0.0001$ (Table III). As shown in Fig. 1, the expression levels of 8 genes were increased and those of the remaining 6 were decreased in the responder group as compared to the non-responder group. A supervised hierarchical clustering analysis using this set of genes with Cluster and Treeview software (http://rana.lbl.gov/ EisenSoftware.htm) yielded good separation of the two groups with regard to the response to GC treatment (Fig. 1).

Establishment of prediction scoring system for clinical response to GC neoadjuvant chemotherapy. Using the 14-gene set that seemed to distinguish the two groups, we calculated the prediction score of each sample by the weighted-vote method (12). Subsequently, we rank-ordered these candidates on the basis of the significance of their permutation P-values (Table III) and calculated prediction scores by the leave-one-out cross-validation test. For the leave-one-out test, we withheld one sample and calculated the permutation P-values and the mean expression levels using the remaining samples to identify genes that most powerfully separated the responder group from the non-responder group. As shown in Table III, importin 7 (IPO7) and hypothetical LOC613266 (LOC613266) revealed a $\mathrm{P}$-value of $<10^{-6}$ in the permutation test $\left(\mathrm{P}=3.96 \times 10^{-7}\right.$ and $\mathrm{P}=8.38 \times 10^{-7}$, respectively). IPO7, which was up-regulated in the tumors belonging to the nonresponder group, imports proteins into the nucleus by acting as an adapter-like protein (16) and is reported to mediate the 
Responders Non-responders

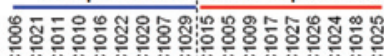

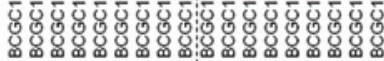

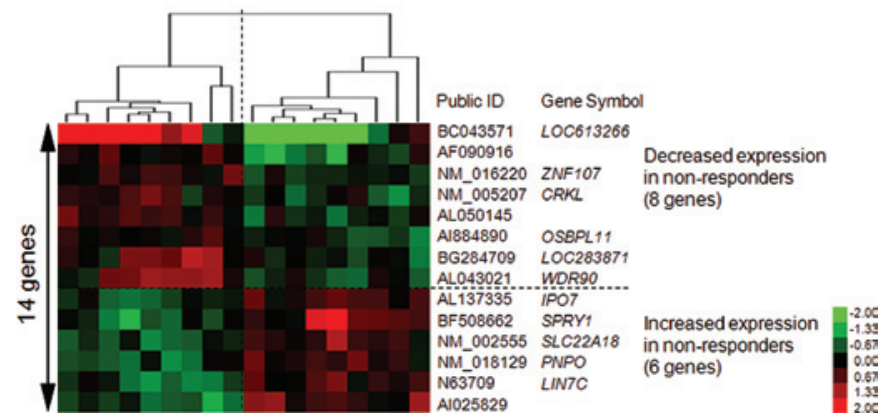

Figure 1. Expression patterns of the 14 genes that discriminated the 9 responders from the 9 non-responders among the bladder cancer patients treated with GC. Horizontal rows represent individual genes; vertical columns represent individual samples. Each cell in the matrix represents the expression level of a single transcript in a single sample, with red and green indicating transcript levels, respectively, above and below the median for that gene across all samples. Black represents unchanged expression or slight expression (intensities of signal under the cutoff value). Color saturation is proportional to the magnitude of the difference.

penetration of the interacting extracellular signal-regulated kinases (ERK) into the nucleus through nuclear pores (16). Since the ERK signaling pathway was reported to be in part responsible for the resistance to gemcitabine in hepatocellular, pancreatic and cholangiocellular carcinomas $(17,18)$, up-regulated expression of IPO7 may contribute to resistance to gemcitabine-combined therapy for bladder cancer through the ERK signaling pathway.

We calculated the classification score (CS) using the prediction scores of the 9 responders and 9 non-responders in various combinations of selected genes and obtained the best separation of the two groups by using the 12 genes that were ranked highest in our candidate gene list (Fig. 2A and B; Table IV). A hierarchical clustering analysis using this set of genes with Cluster and Treeview software yielded good separation of the two groups with regard to sensitivity to the GC neoadjuvant chemotherapy (Fig. 2C).

Furthermore, to verify the prediction scoring system based on the expression data for this set of 12 genes, we examined 19 'test' cases (11 responders and 8 non-responders; Fig. 2B). We investigated gene expression profiles in each of the 19 test cases and then calculated the prediction scores. As shown in Fig. 2B, for 18 of the 19 test cases, the clinical responses were correctly predicted according to the calculated prediction scores. Our data suggest that expression levels of these 12 genes or a subset may play important roles in cellular responses induced by GC neoadjuvant chemotherapy.

Establishment of a quantitative reverse transcription-PCRbased prediction scoring system. To further validate the results of the microarray analysis, we carried out real-time quantitative RT-PCR for the 12 predictive genes and one quantitative control gene, CCT6A, using the 32 cases (14 learning and 18 test cases) (13). We observed significant concordance between the results from the microarray and those of the quantitative RT-PCR experiments. As shown in Table V, Pearson and

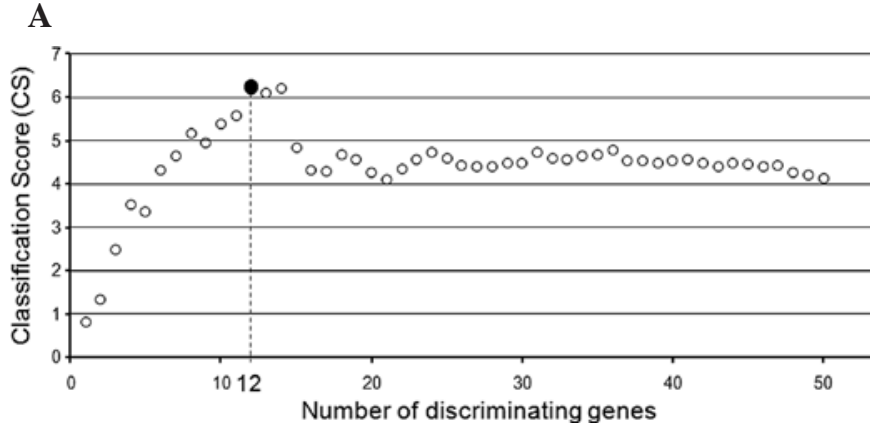

B

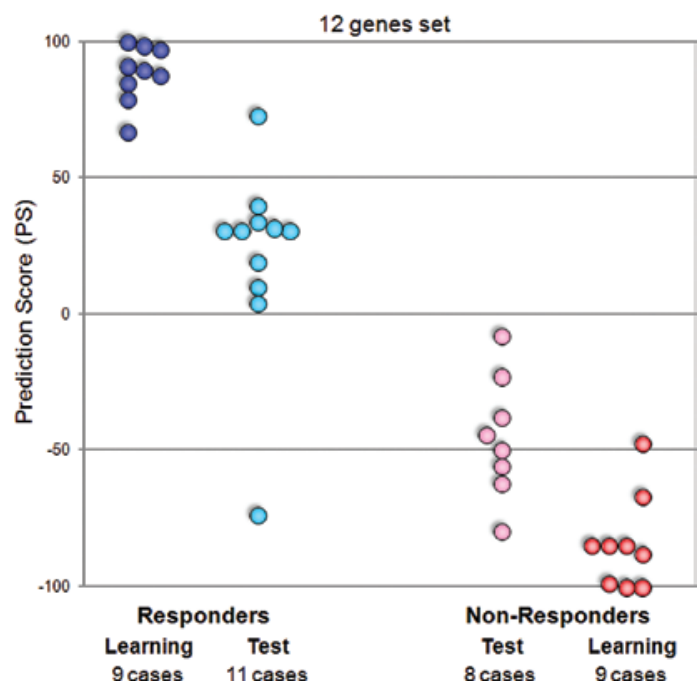

C

Responders Non-responders

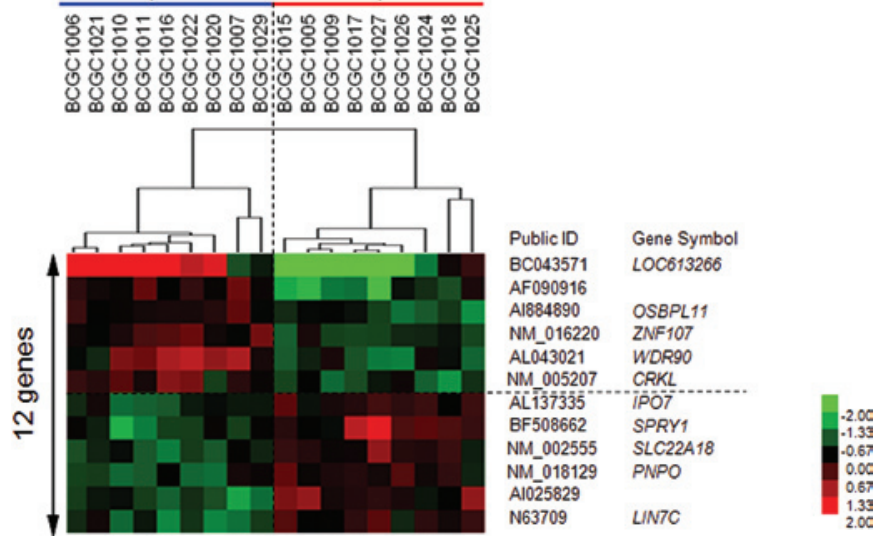

Figure 2. (A) Optimization of the number of discriminating genes. The classification score (CS) was calculated using the prediction scores of the responders $\left(\mathrm{PS}_{\mathrm{r}}\right)$ and non-responders $\left(\mathrm{PS}_{\mathrm{n}}\right)$ in each gene set as follows: $\mathrm{CS}=\left[\mu\left(\mathrm{PS}_{\mathrm{r}}\right)-\mu\left(\mathrm{PS}_{\mathrm{n}}\right)\right] /\left[\delta\left(\mathrm{PS}_{\mathrm{r}}\right)+\delta\left(\mathrm{PS}_{\mathrm{n}}\right)\right]$. A larger value for CS indicates better separation of the two groups by the prediction scoring system. (B) Blue and red circles indicate scores for cross-validation cases of patients whose expression data were used for selecting discriminating genes (learning). Light blue and pink circles represent scores for the 19 additional cases (test). (C) Clustering analysis of 12 predictive genes. All samples fell appropriately into one of two 'trees' according to their sensitivity to GC neoadjuvant chemotherapy.

Spearman rank correlations were positive and statistically significant for all of the genes.

Hence, we attempted to adapt our prediction system on the basis of quantitative real-time RT-PCR to apply to the clinical test. We performed quantitative real-time RT-PCR of 12 predictive genes for 14 learning and 18 test cases, and calculated the prediction scores for all cases. When we esti- 
Table IV. List of the 12 predictive genes.

\begin{tabular}{lll}
\hline Public ID & Gene symbol & \\
\hline AL137335 & IPO7 & Gene name \\
BC043571 & LOC613266 & Importin 7 \\
BF508662 & SPRY1 & Sprothetical LOC613266 homolog 1, antagonist of FGF signaling (Drosophila) \\
AI884890 & OSBPL11 & Oxysterol binding protein-like 11 \\
NM_016220 & ZNF107 & Zinc finger protein 107 \\
AI025829 & & CDNA clone IMAGE:5287121 \\
AF090916 & & Clone HQ0312 \\
N63709 & LIN7C & Lin-7 homolog C (C. elegans) \\
AL043021 & WDR90 & WD repeat domain 90 \\
NM_002555 & SLC22A18 & Solute carrier family 22, member 18 \\
NM_018129 & PNPO & Pyridoxamine 5'-phosphate oxidase \\
NM_005207 & CRKL & V-crk sarcoma virus CT10 oncogene homolog (avian)-like \\
\hline
\end{tabular}

Table V. Correlation of microarray expression data with quantitative-PCR-derived values.

\begin{tabular}{|c|c|c|c|c|c|}
\hline Public ID & Gene symbol & Pearson's correlation coefficient & P-value & Spearman's rank correlation & P-value \\
\hline AL137335 & IPO7 & 0.67 & $2.6 \times 10^{-5}$ & 0.69 & $2.4 \times 10^{-5}$ \\
\hline ВC043571 & LOC613266 & 0.88 & $6.9 \times 10^{-9}$ & 0.96 & $2.5 \times 10^{-6}$ \\
\hline BF508662 & $S P R Y 1$ & 0.85 & $9.4 \times 10^{-10}$ & 0.86 & $2.7 \times 10^{-7}$ \\
\hline AI884890 & OSBPL11 & 0.73 & $2.7 \times 10^{-6}$ & 0.74 & $2.9 \times 10^{-6}$ \\
\hline NM_016220 & ZNF107 & 0.82 & $1.0 \times 10^{-8}$ & 0.78 & $1.1 \times 10^{-6}$ \\
\hline AI025829 & & 0.47 & $6.2 \times 10^{-3}$ & 0.50 & $4.0 \times 10^{-3}$ \\
\hline AF090916 & & 0.84 & $2.0 \times 10^{-9}$ & 0.86 & $2.5 \times 10^{-7}$ \\
\hline N63709 & LIN7C & 0.81 & $1.9 \times 10^{-8}$ & 0.74 & $3.1 \times 10^{-6}$ \\
\hline AL043021 & WDR90 & 0.79 & $7.1 \times 10^{-8}$ & 0.79 & $9.1 \times 10^{-7}$ \\
\hline NM_002555 & SLC22A18 & 0.85 & $8.1 \times 10^{-10}$ & 0.84 & $4.3 \times 10^{-7}$ \\
\hline NM_018129 & PNPO & 0.70 & $7.4 \times 10^{-6}$ & 0.70 & $1.7 \times 10^{-5}$ \\
\hline NM_005207 & $C R K L$ & 0.54 & $1.3 \times 10^{-3}$ & 0.60 & $3.3 \times 10^{-4}$ \\
\hline
\end{tabular}

mated these scores by the leave-one-out cross-validation test, all learning cases and 17 of the 18 test cases were categorized correctly according to their response to GC neoadjuvant chemotherapy (Fig. 3).

Clinical implication of the two systems that predict the response to GC and M-VAC therapy. We previously reported a system with nearly $90 \%$ accuracy to predict the clinical response to the M-VAC regimen, a combination of methotrexate, vinblastine, doxorubicin and cisplatin neoadjuvant chemotherapy, for patients with invasive bladder cancer (13). To investigate how patients treated with GC therapy respond to M-VAC therapy, we simulated the clinical response of the $37 \mathrm{GC}$-treated patients to the M-VAC therapy using our prediction system for M-VAC neoadjuvant chemotherapy (13). As shown in Fig. 4A, 14 of the 19 cases predicted to be $\mathrm{GC}$ responders were suspected to be responders to M-VAC therapy, and 11 of 18 cases predicted to be GC non-responders were suspected to be responders to M-VAC therapy according to our M-VAC prediction scoring system. On the other hand, we applied the scoring system to the GC prediction system indicated above to the 39 M-VAC-treated patients that had been reported previously (13). Seven of the 25 cases predicted to be M-VAC responders were calculated to be responders to GC therapy, and 8 of the 14 cases predicted to be M-VAC non-responders were calculated to be responders to $\mathrm{GC}$ therapy (Fig. 4B). The distribution of the cases according to the predicted response to GC or M-VAC therapy is summarized in Fig. 4C. Among the the 76 patients treated with either GC or M-VAC, 21 patients were predicted to be responders of both GC and M-VAC therapies, 13 were predicted to be responders to GC therapy, 29 were predicted to be responders to M-VAC therapy and 13 were likely to be non-responders to both therapies (Fig. 4C). Sixty-three of the 76 patients could be expected to respond to both or either of these two regimens by applying our two prediction systems. However, the positive and negative predictive accuracies of the prediction systems for response to $\mathrm{M}-\mathrm{VAC}\left(\mathrm{PPA}_{\mathrm{MVAC}}, \mathrm{NPA}_{\mathrm{MVAC}}\right)$ and $\mathrm{GC}\left(\mathrm{PPA}_{\mathrm{GC}}, \mathrm{NPA}_{\mathrm{GC}}\right)$ were: $\mathrm{PPA}_{\mathrm{MVAC}}=14 / 16, \mathrm{NPA}_{\mathrm{MVAC}}=$ $5 / 5$ (data not shown), $\mathrm{PPA}_{\mathrm{GC}}=10 / 10$ and $\mathrm{NPA}_{\mathrm{GC}}=8 / 9$ (Fig. 


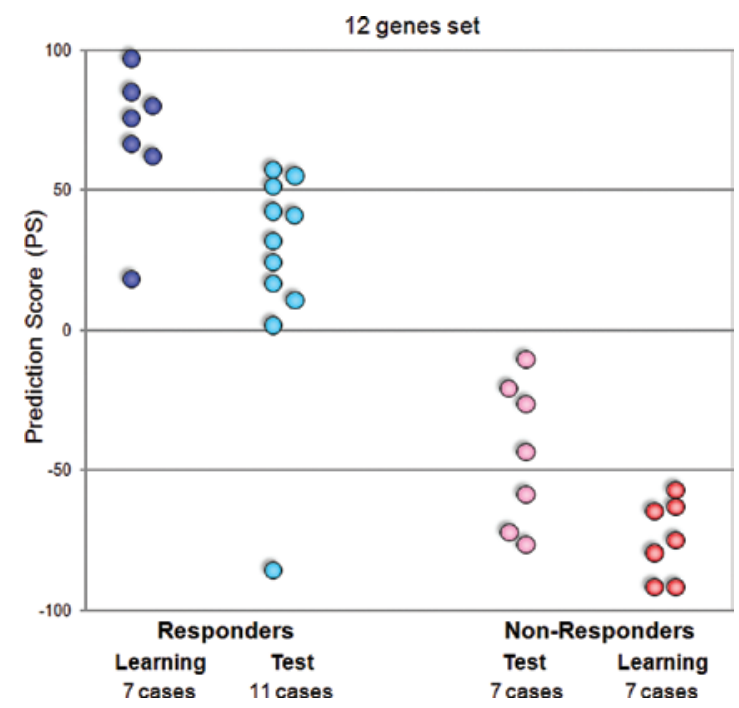

Figure 3. Quantitative RT-PCR-based prediction scoring system. Prediction scores for 32 cases using values derived from quantitative RT-PCR experiments of 12 predictive genes. Blue and red circles indicate scores for selecting discriminating genes (learning cases). Light blue and pink circles represent scores of 18 additional (test) cases.

2B). Hence, $80.1 \%\left(1-\left[13 \times \mathrm{NPA}_{\mathrm{GC}} \times \mathrm{NPA}_{\mathrm{MVAC}}+29 \times \mathrm{NPA}_{\mathrm{GC}}\right.\right.$ $\left.\left.\mathrm{x}\left(1-\mathrm{PPA}_{\mathrm{MVAC}}\right)\right] / 76\right)$ of the 76 patients were calculated to be responders to GC and/or M-VAC therapies (see Materials and methods) by the combination of the two systems. Without these types of prediction system, the responders to GC and M-VAC therapy were limited to $54.1 \%$ (20 of 37 cases) and $59.0 \%$ (23 of 39 cases), respectively (Fig. 4). Since patients usually are able to undergo neoadjuvant treatment once, the application of these prediction scores should improve the quality of life of cancer patients.

\section{Discussion}

Microarray analysis is now widely applied to examine the expression of thousands of genes simultaneously in cancer cells. However, in the great majority of previous reports, adequate attention has not been paid to the quality of the materials and experiments. For example, clinical samples (surgically resected tissues or biopsy materials) usually consist of various cellular components, and the proportions of cancer cells in given individual tissues vary enormously from one tumor to another (19). To obtain precise expression data of cancer cells, we applied a laser microbeam microdissection system to enrich as much as possible the populations of cancer cells from the biopsy specimens of 37 invasive bladder cancers in order to establish a scoring system to predict response to GC therapy.

Despite recent advances, approximately $50 \%$ of patients with bladder cancer who receive GC chemotherapy show no or very poor response in terms of staging, and a large proportion of them suffer from adverse events, such as myelosuppression and/or gastrointestinal toxicity $(9,10)$. Although certain factors have been reported to be associated with chemosensitivity or prognosis of bladder cancer patients (20-24), characterization of tumor features using only one or a few of these factors has failed thus far to reliably predict individual responses, indicating a need for a more accurate method for predicting response to anticancer drugs. This study was designed to develop a prediction system for GC neoadjuvant chemotherapy on the basis of gene expression profiles of purified populations of bladder cancer cells. We identified 14 genes whose expression was significantly different between the responders and non-responders and further ranked them by statistical significance of the permutation test $(\mathrm{P}<0.0001$; Table III). Then, we further selected 12 genes and established the numerical scoring system. Moreover, we tested the scoring
$\mathbf{A}$

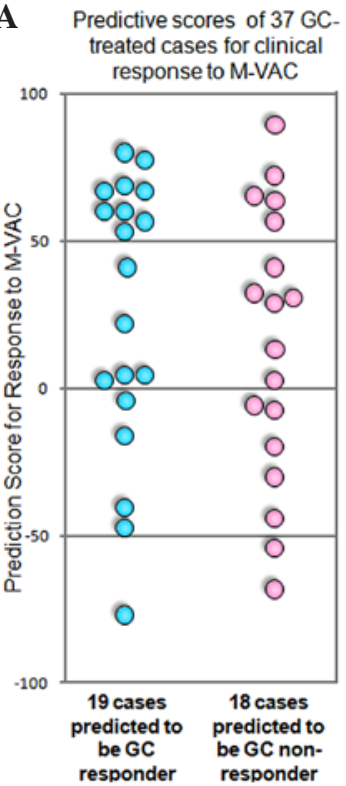

B

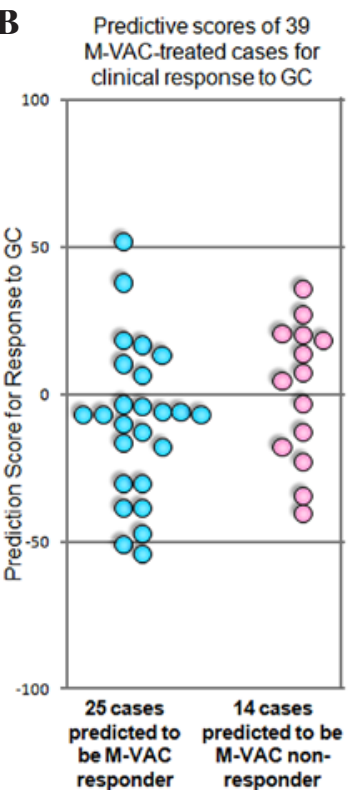

$\mathbf{C}$

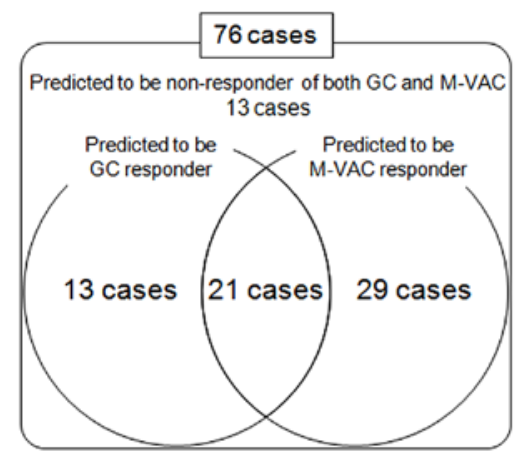

Figure 4. (A) Distribution of the prediction scores of 37 GC-treated patients for clinical response to M-VAC chemotherapy. Light blue and pink circles represent cases predicted to be GC responders and non-responders, respectively. (B) Distribution of prediction scores of 39 M-VAC-treated patients for clinical response to GC chemotherapy. Light blue and pink circles represent cases predicted to be M-VAC responders and non-responders, respectively. (C) Venn diagram indicating the distribution of the 76 cases according to the response to GC or M-VAC therapy. 
system by the leave-one-out cross-validation method and found it to provide the best separation of the responders from the non-responders. Furthermore, our scoring system was able to predict accurately the response of 18 of the 19 additional test cases to GC neoadjuvant chemotherapy (Fig. 2B).

The list of 14 genes that showed significant differences between the two groups might provide insight into the biological mechanism(s) underlying sensitivity or resistance to GC chemotherapy. Among those 14 genes, IPO7, which imports protein into the nucleus by acting as an adapter-like protein, was up-regulated in the non-responders (Fig. 1, Table III). Although hypoxia inducible factor $1, \alpha$ subunit (HIF-1 $\alpha$ ), which is one of the substrates of IPO7, is known to activate the connective tissue growth factor/cysteine-rich 61 (CCN1) (25), CCN1 was found to confer resistant to carboplatin or cisplatininduced apoptosis by inhibiting caspase-3 activity on ovarian cancer cells $(25,26)$. Therefore, up-regulated expression of IPO7 may contribute to resistance to GC neoadjuvant chemotherapy through inhibiting caspase-3 activity. Furthermore, solute carrier family 22 , member 18 (SLC22A18), which is a polyspecific organic cation transporter, was up-regulated in the non-responders. This gene encodes a predicted protein with multiple membrane-spanning segments that belongs to the polyspecific transporter/multi-drug resistance gene family (27). The pharmacogenomic approach based on the correlation of expression and sensitivity data sets derived from the NCI-60 cell panel suggest that SLC22A18 could be a transporter of gemcitabine (28). Hence, up-regulated expression of SLC22A18 may contribute to resistance to GC neoadjuvant chemotherapy by the efflux of gemcitabine from cancer cells through the transporter.

Previously, other research groups have predicted the prognosis or chemosensitivity of tumors based on quantitive RT-PCR results for the expression of genes selected through microarray analysis (29,30). To confirm the reliability of microarray data and open the possibility of more convenient prediction strategies for routine clinical use, we also performed quantitive RT-PCR experiments using the 12 predictive genes and the 32 learning and test cases of bladder cancer selected after microarray analysis. We confirmed a significant correlation between the data obtained for the 32 paired samples upon microarray and results of quantitive RT-PCR (Table V). Moreover, we verified that our quantitative RT-PCR-based prediction system could also correctly classify 17 of the 18 subsequent test cases with regard to their drug response (Fig. 3).

We previously reported a scoring system to predict response to M-VAC therapy, applying a laser microbeam microdissection system (13). In the present study, in order to simulate how patients treated with GC or M-VAC therapy respond to M-VAC or GC therapy, we calculated their prediction scores for the response to M-VAC or GC therapy using the two prediction systems, respectively. As shown in Fig. 4, among the 76 patients, only 21 and 13 cases were expected to be responders and non-responders to both GC and M-VAC therapies, respectively, suggesting that the sensitivity or resistance to M-VAC therapy was unlikely to be correlated with the sensitivity or resistance to GC therapy (Fig. 4C). Although a number of patients with invasive bladder cancer have received neoadjuvant chemotherapies, such as M-VAC or GC without prediction of their responses, the response rate (complete or partial response) to either of the GC or M-VAC therapy has been reported to be approximately $50 \%$ (7-10). Since our present and previous studies indicated that positive predictive accuracies for M-VAC and GC were $87.5 \%$ (14/16; data not shown) and $100 \%$ (10/10), respectively, personalized selection of an appropriate chemotherapy with a combination of the two prediction systems would be expected to improve the response rate to the chemotherapies. In addition, if all patients with invasive bladder cancer were treated with appropriate therapy based on the results of prediction systems, $80.1 \%$ would achieve significant tumor shrinking $(>60 \%)$ by either or both of the two regimens.

In conclusion, we imply with some confidence that our prediction system for the sensitivity of invasive bladder cancers to GC therapy as well as M-VAC therapy, which was reported previously on the basis of either the microarrayderived expression profiles or the quantitative RT-PCR results, should provide opportunities for achieving better prognosis and improved quality of life for patients, leading to higher response rate of neoadjuvant chemotherapy, although a larger scale study is certainly warranted. Moreover, appropriate neoadjuvant chemotherapy for each patient with invasive bladder cancer using these systems might encourage minimal surgery for invasive bladder cancer. Our data suggest that the goal of 'personalized medicine', prescribing the correct treatment regimen for each patient, may be achievable by selecting specific sets of genes for their predictive values according to the approach demonstrated here.

\section{Acknowledgements}

We thank Kumi Matsuda for the real-time RT-PCR experiments, Noriko Sudo for the management of clinical tissue, Noriko Ikawa for the preparation of tissue sections and Takashi Morizono for the analysis of data. We also thank all urologists of the Iwate Medical University for providing patient samples and clinical information.

\section{References}

1. Parkin DM, Bray F, Ferlay J and Pisani P: Global cancer statistics, 2002. CA Cancer J Clin 55: 74-108, 2005.

2. Sternberg CN: The treatment of advanced bladder cancer. Ann Oncol 6: 113-126, 1995.

3. Fagg SL, Dawson-Edwards P, Hughes MA, Latief TN, Rolfe EB and Fielding JW: Cis-diamminedichloroplatinum (DDP) as initial treatment of invasive bladder cancer. Br J Urol 56: 296-300, 1984.

4. Raghavan D, Pearson B, Coorey G, et al: Intravenous cis-platinum for invasive bladder cancer. Safety and feasibility of a new approach. Med J Aust 140: 276-278, 1984.

5. Advanced Bladder Cancer Meta-analysis Collaboration: Neoadjuvant chemotherapy in invasive bladder cancer: a systematic review and meta-analysis. Lancet 361: 1927-1934, 2003.

6. Grossman HB, Natale RB, Tangen CM, et al: Neoadjuvant chemotherapy plus cystectomy compared with cystectomy alone for locally advanced bladder cancer. N Engl J Med 349: 859-866, 2003.

7. Von der Maase H, Sengelov L, Roberts JT, et al: Long-term survival results of a randomized trial comparing gemcitabine plus cisplatin, with methotrexate, vinblastine, doxorubicin, plus cisplatin in patients with bladder cancer. J Clin Oncol 23: 4602-4608, 2005.

8. Cam K, Yildirim A, Ozveri H, Turkeri L and Akdas A: The efficacy of neoadjuvant chemotherapy in invasive bladder cancer. Int Urol Nephrol 33: 49-52, 2002. 
9. Dogliotti L, Carteni G, Siena S, et al: Gemcitabine plus cisplatin versus gemcitabine plus carboplatin as first-line chemotherapy in advanced transitional cell carcinoma of the urothelium: results of a randomized phase 2 trial. Eur Urol 52: 134-141, 2007.

10. Bamias A, Moulopoulos LA, Koutras A, et al: The combination of gemcitabine and carboplatin as first-line treatment in patients with advanced urothelial carcinoma. A Phase II study of the Hellenic Cooperative Oncology Group. Cancer 106: 297-303, 2006.

11. Nikolova DN, Zembutsu H, Sechanov T, et al: Genome-wide gene expression profiles of thyroid carcinoma: identification of molecular targets for treatment of thyroid carcinoma. Oncol Rep 20: 105-121, 2008.

12. Golub TR, Slonim DK, Tamayo P, et al: Molecular classification of cancer: class discovery and class prediction by gene expression monitoring. Science 286: 531-537, 1999.

13. Takata R, Katagiri T, Kanehira M, et al: Predicting response to methotrexate, vinblastine, doxorubicin, and cisplatin neoadjuvant chemotherapy for bladder cancers through genome-wide gene expression profiling. Clin Cancer Res 11: 2625-2636, 2005.

14. Yamanaka Y, Tamari M, Nakahata T and Nakamura Y: Gene expression profiles of human small airway epithelial cells treated with low doses of 14- and 16-membered macrolides. Biochem Biophys Res Commun 287: 198-203, 2001.

15. Takata R, Katagiri T, Kanehira M, et al: Validation study of the prediction system for clinical response of M-VAC neoadjuvant chemotherapy. Cancer Sci 98: 113-117, 2007.

16. Zehorai E, Yao Z, Plotnikov A and Seger R: The subcellular localization of MEK and ERK - a novel nuclear translocation signal (NTS) paves a way to the nucleus. Mol Cell Endocrinol 314: 213-220, 2009.

17. Matsumoto K, Nagahara T, Okano J and Murawaki Y: The growth inhibition of hepatocellular and cholangiocellular carcinoma cells by gemcitabine and the roles of extracellular signal-regulated and checkpoint kinases. Oncol Rep 20: 863-872, 2008.

18. Yokoi K and Fidler IJ: Hypoxia increases resistance of human pancreatic cancer cells to apoptosis induced by gemcitabine. Clin Cancer Res 10: 2299-2306, 2004.
19. Iwao-Koizumi K, Matoba R, Ueno N, et al: Prediction of docetaxel response in human breast cancer by gene expression profiling. J Clin Oncol 23: 422-431, 2005.

20. Esrig D, Elmajian D, Groshen S, et al: Accumulation of nuclear p53 and tumor progression in bladder cancer. N Engl J Med 331: 1259-1264, 1994

21. Cote RJ, Dunn MD, Chatterjee SJ, et al: Elevated and absent $\mathrm{pRb}$ expression is associated with bladder cancer progression and has cooperative effects with p53. Cancer Res 58: 1090-1094, 1998.

22. Oosterhuis JW, Schapers RF, Janssen-Heijnen ML, Smeets AW and Pauwels RP: MIB-1 as a proliferative marker in transitional cell carcinoma of the bladder: clinical significance and comparison with other prognostic factors. Cancer 88: 2598-2605, 2000.

23. Stein JP, Ginsberg DA, Grossfeld GD, et al: Effect of p21WAF1/ CIP1 expression on tumor progression in bladder cancer. J Natl Cancer Inst 90: 1072-1079, 1998.

24. Inoue K, Slaton JW, Karashima T, et al: The prognostic value of angiogenesis factor expression for predicting recurrence and metastasis of bladder cancer after neoadjuvant chemotherapy and radical cystectomy. Clin Cancer Res 6: 4866-4873, 2000

25. Rho SB, Woo JS, Chun T and Park SY: Cysteine-rich 61 (CYR61) inhibits cisplatin-induced apoptosis in ovarian carcinoma cells. Biotechnol Lett 31: 23-28, 2009.

26. Gery S, Xie D, Yin D, et al: Ovarian carcinomas: CCN genes are aberrantly expressed and $\mathrm{CCN} 1$ promotes proliferation of these cells. Clin Cancer Res 11: 7243-7254, 2005.

27. Dao D, Frank D, Qian N, et al: IMPT1, an imprinted gene similar to polyspecific transporter and multi-drug resistance genes. Hum Mol Genet 7: 597-608, 1998

28. Okabe M, Szakacs G, Reimers MA, et al: Profiling SLCO and SLC22 genes in the NCI-60 cancer cell line to identify drug uptake transporters. Mol Cancer Ther 7: 3081-3091, 2008.

29. Lossos IS, Czerwinski DK, Alizadeh AA, et al: Prediction of survival in diffuse large-B-cell lymphoma based on the expression of six genes. N Engl J Med 350: 1828-1837, 2004.

30. Ma XJ, Wang Z, Ryan PD, et al: A two-gene expression ratio predicts clinical outcome in breast cancer patients treated with tamoxifen. Cancer Cell 5: 607-616, 2004. 